\title{
Evaluation of the Physical and Adhesive Properties of Natural Weathering Asphalt
}

\author{
Yanzhu Wang $\mathbb{D}^{1},{ }^{1}$ Xudong Wang $\mathbb{D}^{1,2}$ Xingye Zhou, ${ }^{2}$ Guang Yang, ${ }^{1}$ and Lei Zhang ${ }^{2}$ \\ ${ }^{1}$ School of Transportation Science and Engineering, Harbin Institute of Technology, Harbin 150090, China \\ ${ }^{2}$ Research Institute of Highway, Ministry of Transport, Beijing 100088, China \\ Correspondence should be addressed to Xudong Wang; xd.wang@rioh.cn
}

Received 23 July 2020; Revised 5 November 2020; Accepted 16 December 2020; Published 2 January 2021

Academic Editor: Meng Guo

Copyright ( 2021 Yanzhu Wang et al. This is an open access article distributed under the Creative Commons Attribution License, which permits unrestricted use, distribution, and reproduction in any medium, provided the original work is properly cited.

Asphalt suffers from a series of aging processes in the natural environment. This is a significant factor in asphalt pavement diseases. Research of the properties of the decay processes in the natural weathering of asphalt will be helpful in distinguishing the characteristics of the various types of asphalt and in the selection of pavement materials. Neat asphalt A70, a styrene-butadienestyrene- (SBS-) modified asphalt, and crumb-rubber-modified asphalt AR are exposed to outdoor conditions to weather naturally. This process is traced by testing the basic physical properties and the surface free energy of asphalts, using the sessile drop method. Results illustrate that the basic physical properties of asphalt change significantly during the natural weathering process and that the rubber asphalt has the superior aging resistance, while the neat asphalt A70 has a high aging susceptibility. Furthermore, the presence of the SBS and rubber-powder modifiers transforms the change trend of the surface free energy of neat asphalt. The adhesion work between aggregate and asphalt can be used to quantitatively evaluate the adhesive properties between them. Meanwhile, the presence of moisture between the asphalt and aggregate changes cohesive failure into adhesive failure.

\section{Introduction}

In the natural environment, asphalt will undergo complex physical and chemical reactions under the influence of sunlight, oxygen, and heat and lead to the deterioration of asphalt performance [1-3]. The adhesion failure of asphalt that manifests as the peeling off from the aggregate surface (caused by the presence of moisture) is another important factor that affects the durability of the asphalt pavement [4-6]. Thus, understanding the physical and adhesive properties of the naturally weathering asphalt is crucial.

Currently, research on the simulation of the aging of asphalt is widespread. Most of this research is carried out in laboratories. Aging methods include the thin-film oven test (TFOT), the rolling TFOT, and the pressurized aging vessel [7]. These standard laboratory-aging methods are the shortterm and long-term aging simulations of asphalt [8-11]. To simulate natural weathering conditions optimally, researchers evaluate the performance of asphalt after accelerated weather aging. An asphalt sample with a certain thickness is irradiated for a period of time at a designated temperature and with an irradiance of ultraviolet radiation intensity [12-14]. However, the methods of the laboratory simulation of the asphalt sample still cannot reflect the natural environment fully. Thus, to study the real situation of asphalt aging in the natural environment, some researchers are exposing modified asphalts to outdoor conditions to subject them to natural weathering for one year at most $[2,15,16]$. This is insufficient time for achieving a simulation of the entire service life of asphalt exposed to outdoor conditions.

Asphalt-aging research aims mainly to establish performance indicators to quantify the aging-degree of asphalt in order to improve predictions of the properties of asphalt. At present, the traditional evaluation indexes of asphaltaging performance include basic physical properties (e.g., penetration, softening point, and ductility) and rheological indicators (e.g., viscosity, complex modulus $(G *)$, and phase angle $(\delta)$ ) [7]. Many modern test methods, such as the atom force microscope (AFM), have been applied. AFM has 
the potential to measure adhesion differences among micron-sized domains in asphalt binders [17]. Yu et al. evaluated the adhesive property of aging asphalt binders quantitatively by using the AFM method [18]. Research shows that asphalt pavement performance is related to the cohesive and adhesive bonding within the asphalt-aggregate system, and the cohesive-adhesive bonding is related to the surface free energy characteristics of the system [19]. However, studies of the changes of cohesive and adhesive properties of asphalt after natural weathering (based on surface free energy theory) are few.

Generally, traditional and advanced performance indicators can realize the evaluation and prediction of asphaltaging performance by standard laboratory aging methods and by self-designed aging methods. However, research on the natural weathering of asphalt in regard to aging methods is still insufficient, and the allowed natural-aging times are not long enough. However, studies of the cohesive and adhesive properties of asphalt after natural weathering (based on surface free energy theory) are significant.

To evaluate the physical and adhesive properties of naturally weathered asphalt, three kinds of asphalt are exposed to natural weathering. Every year, samples are retrieved for performance evaluation. At this point, they have been aging for 4 years. The performance indicators include penetration, softening point, ductility, and viscosity. The surface free energy of the asphalt samples is investigated at various aging times.

\section{Materials and Experiment Design}

2.1. Materials. Neat asphalt $\mathrm{A} 70$ with penetration grade 70 and SBS-modified asphalt (SBSMA) were used in this study. Three kinds of rubber asphalt were made, using the "wet process." The performance indexes of rubber asphalt are shown in Table 1. According to the "Specification for Asphalt Rubber Pavement and Construction" provisions in China, the elastic recovery rate shall not be less than $70 \%$ when the softening point is greater than $60^{\circ} \mathrm{C}$. Finally, rubber asphalt with 22 wt. \% crumb-rubber-powder content was used. The PG grades of A70, SBSMA, and AR-22\% were PG64-22, PG82-22, and PG82-28, respectively. The aggregates used were limestone, basalt, and granite.

2.2. Aging Procedure. A certain amount of asphalt was weighed and poured on folded and fixed-size silver paper. Then, the silver paper with its asphalt load was placed on a glass plate and then into the oven. The glass plate was adjusted with a level ruler to be horizontal. The oven temperature was controlled at $100^{\circ} \mathrm{C}$ and $135^{\circ} \mathrm{C}$, so that the base and modified asphalts distribute evenly, such that the thickness of the asphalt film is $2 \mathrm{~mm}$. Finally, all the samples were exposed to outdoor conditions-in this case, to the weather in Beijing City, a temperate-zone monsoon climate area-to age in the natural environment (see Figure 1). The weather-aging was carried out over 4 years, from January 2016 to January 2020 . The asphalts were retrieved for testing at yearly intervals.

\subsection{Characterization Methods}

2.3.1. Basic Performance Test. Penetration, softening point, and ductility were measured according to ASTM D5, ASTM D36, and ASTM D113, respectively. Viscosity was obtained by using a Brookfield viscometer. Approximately $8.5 \mathrm{~g}$ of sample was placed into a Brookfield viscometer test tube and tested with a spindle rotating at $20 \mathrm{rpm}$, for neat asphalt and SBSMA (ASTM D4402) [7]. The torque was controlled at $50 \%$ in the viscosity test for AR, and the stretching speed in the ductility test for AR asphalt was $1 \mathrm{~cm} / \mathrm{min}$, in accordance with the provisions of the "Guidelines for Design and Construction of Asphalt Rubber and Mixture in Beijing" (2006).

2.3.2. Contact Angle Test. To calculate the surface energy of asphalt, the contact angle of the standard liquid with known surface-energy parameters on the asphalt surface must be measured. In this research, the contact angle was measured by the sessile drop method on a drop-shape analysis instrument made in Germany. The sessile drop method schematic is shown in Figure 2.

According to the theory of van Oss et al., the total surface free energy can be divided into two components, namely, the nonpolar Lifshitz-van der Waals (LW) component and the polar Lewis acid-base (AB) component [20].

$$
\gamma=\gamma^{d}+\gamma^{p}
$$

where $\gamma$ is the surface free energy of the solid material, $\gamma^{d}$ is the dispersive component of surface free energy, and $\gamma^{p}$ is the polarity component of the surface free energy.

The contact angle $(\theta)$ can be measured when a liquid $(L)$ is wetted on a solid ( $S$ ) surface, and the interaction energy between $L$ and S can be described by Young's equation as follows [21, 22]:

$$
\gamma_{L} \cos \theta=\gamma_{S}-\gamma_{\mathrm{SL}}
$$

where $\gamma_{L}$ is the surface energy (or surface tension) of the liquid, $\gamma_{S}$ is the surface energy of the solid, and $\gamma_{\mathrm{SL}}$ is the interfacial tension between $L$ and $S$.

According to the theory of interfacial tension, the L-S interface free energy can be expressed as follows [23, 24]:

$$
\gamma_{S L}=\gamma_{S}+\gamma_{L}-2 \sqrt{\gamma_{S}^{d} \gamma_{L}^{d}}-2 \sqrt{\gamma_{S}^{p} \gamma_{L}^{p}}
$$

where $\gamma_{S}^{d}$ is the dispersive component of $\mathrm{S}$ surface free energy, $\gamma_{S}^{p}$ is the polarity component of $S$ surface free energy, $\gamma_{L}^{d}$ is the dispersive component of $L$ surface free energy, and $\gamma_{L}^{p}$ is the polarity component of $L$ surface free energy.

Equation (4) can be obtained by combining equations (2) and (3) as follows:

$$
\gamma_{L}(1+\cos \theta)=2 \sqrt{\gamma_{S}^{d} \gamma_{L}^{d}}+2 \sqrt{\gamma_{S}^{p} \gamma_{L}^{p}}
$$

The transposed form equation (4) is shown as follows: 
TABLE 1: Performance indexes of AR at three contents.

\begin{tabular}{lccc}
\hline $\begin{array}{l}\text { Crumb-rubber } \\
\text { content (\%) }\end{array}$ & $\begin{array}{c}\text { Penetration } \\
0.1 \mathrm{~mm}\left(25^{\circ} \mathrm{C}\right)\end{array}$ & $\begin{array}{c}\text { Softening } \\
\text { point }{ }^{\circ} \mathrm{C}\end{array}$ & $\begin{array}{c}\text { Elastic } \\
\text { recovery rate } \\
\%\end{array}$ \\
\hline 22 & 55.1 & 64.7 & 74.0 \\
20 & 55.8 & 62.1 & 68.9 \\
18 & 56.5 & 60.2 & 65.2 \\
\hline
\end{tabular}

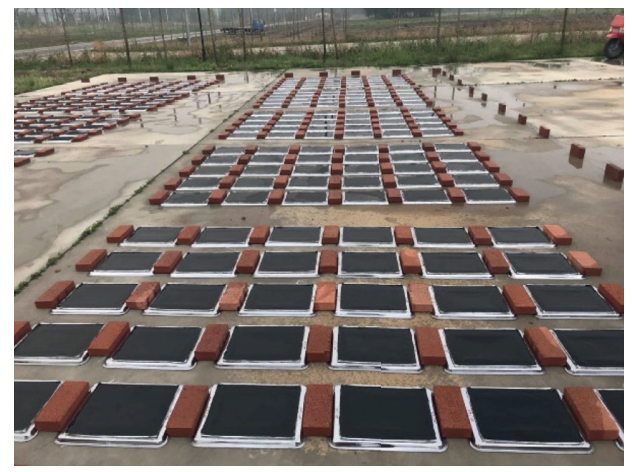

FIGURE 1: Aging environment conditions of natural weathering.

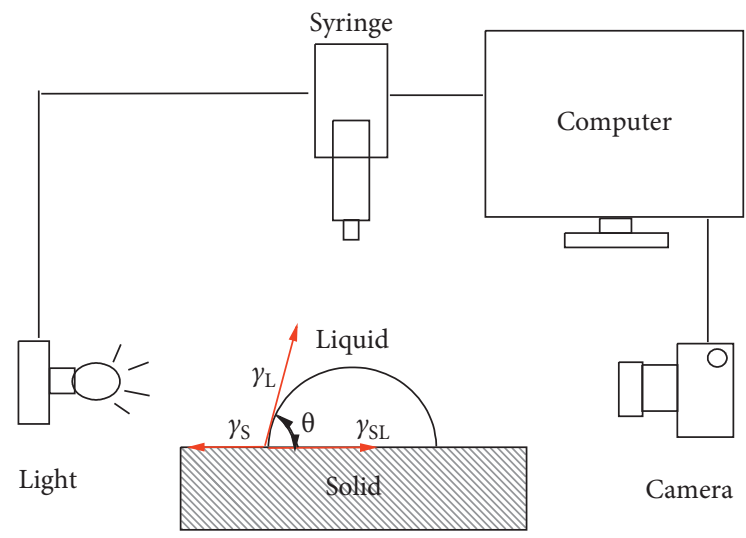

FIgURE 2: Schematic of contact angle measurement.

$$
\frac{(1+\cos \theta) \gamma_{L}}{2 \sqrt{\gamma_{L}^{d}}}=\sqrt{\gamma_{S}^{p}} \sqrt{\frac{\gamma_{L}^{p}}{\gamma_{L}^{d}}}+\sqrt{\gamma_{S}^{d}} .
$$

According to equation (5), the surface energy parameters of $S$ can be obtained by linear analysis when the surface energy parameters of more than two standard reagents are known, and the contact angle on the $S$ surface is measured. In this study, the reagents used to test the surface free energy include distilled water, formamide, glycerol, and ethylene glycol. The surface free energy and components of the reagents are shown in Table 2.

\section{Results and Discussion}

3.1. Basic Physical Properties. The penetration index is used to evaluate the consistency of asphalt. The result can be
TABLE 2: Surface free energy components of reagents [25].

\begin{tabular}{lccc}
\hline Liquids & \multicolumn{3}{c}{$\begin{array}{c}\text { Surface free energy components } \\
\left(\mathrm{mJ} \cdot \mathrm{m}^{-2}\right)\end{array}$} \\
& $\gamma$ & $\gamma^{d}$ & $\gamma^{p}$ \\
\hline Distilled water & 72.30 & 18.70 & 53.60 \\
Formamide & 59.00 & 39.40 & 19.60 \\
Glycerol & 65.20 & 28.30 & 36.90 \\
Ethylene glycol & 48.20 & 29.29 & 18.91 \\
\hline
\end{tabular}

considered a property of the material, and its softness is described. Figure 3 shows the penetration of asphalts of different natural weathering times. The penetration index of asphalt decreases greatly after one year of natural weathering, and the attenuation rate slows down in the three following years. Table 3 presents the residual penetration ratio (penetration ratio after aging and before aging) of asphalt. Among the three kinds of asphalt, the penetration of A70 asphalt decreases the fastest. Its residual penetration ratio is only $39.4 \%$ and $18.5 \%$ after 1 year and after 4 years of natural weathering, respectively. The penetration of AR asphalt decreases the slowest, and its residual penetration ratio is $59.4 \%$ and $37.0 \%$ after 1 year and after 4 years of natural weathering, respectively. The penetration change rate of modified asphalt SBSMA falls between the two. The pavement will be prone to cracking when the penetration value of asphalt at $25^{\circ} \mathrm{C}$ drops below $20(0.1 \mathrm{~mm})$. The penetration value of A70 and SBSMA dropped below 20 in the second and third year, respectively. However, the value of AR is still higher than 20 after four years of natural weathering. This finding indicates that rubber asphalt AR is still soft and difficult to crack after weathering.

The softening point can be used to evaluate the asphalt's susceptibility to heat. Figure 4 presents the softening point of the asphalts with different natural weathering times. Comparison shows that, after the first year of aging, the softening point of asphalt increases the most and rises steadily in the subsequent aging process. The effect of natural weathering on the softening point of neat asphalt A70 is the most significant, but the effect on the modified asphalt is relatively small, and the change rate of the softening point of the two modified asphalts is close. After four years of natural weathering, the softening point change rates of A70, SBSMA, and AR are $48.8 \%, 22.3 \%$, and $26.2 \%$ respectively, as Table 4 shows.

The rheological properties of asphalt are evaluated by viscosity indicator, and its viscosity at $160^{\circ} \mathrm{C}$ is studied. Figure 5 presents the viscosity of the asphalts with different natural weathering times. The viscosity $\left(160^{\circ} \mathrm{C}\right)$ of asphalt increases gradually with the progress of aging. The viscosity change rate of rubber asphalt AR is smaller when compared to the other two kinds of asphalt, and the increase in viscosity after four years of aging is only $45.7 \%$, whereas the viscosity increases of neat asphalt and SBS-modified asphalt reach as high as $432.4 \%$ and $372.9 \%$, respectively, as shown in Table 5. This also shows that viscosity is the most sensitive property index of aging asphalt and that the rubber asphalt has superior aging resistance.

Asphalt ductility is an important performance indicator in evaluating crack resistance. Table 6 presents the ductility dates 


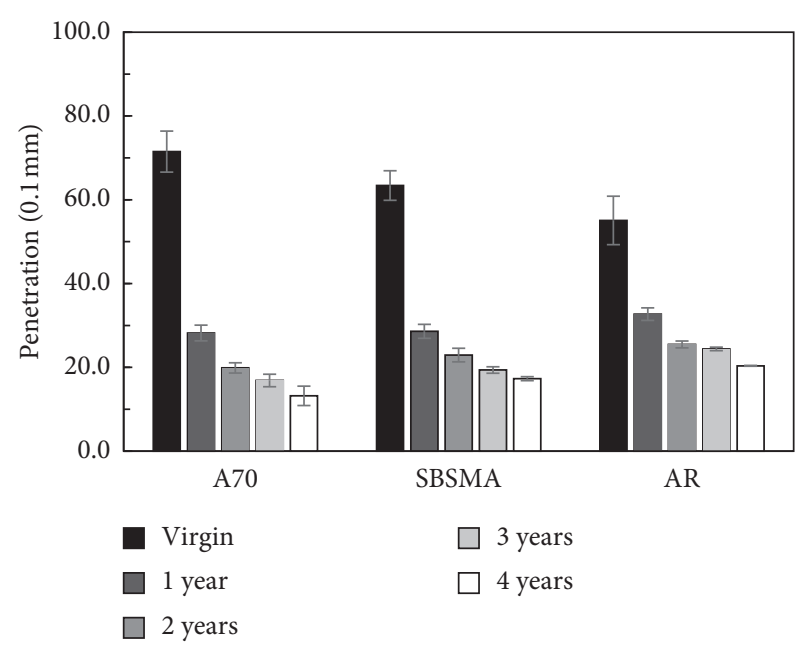

Figure 3: Penetration of asphalt with different weathering time.

TABLE 3: Residual penetration ratio of asphalt.

\begin{tabular}{lcccc}
\hline \multirow{2}{*}{ Asphalt } & \multicolumn{4}{c}{ Residual penetration ratio (\%) } \\
& 1 year & 2 years & 3 years & 4 years \\
\hline A70 & 39.4 & 27.8 & 23.6 & 18.5 \\
SBSMA & 45.1 & 36.2 & 30.5 & 27.3 \\
AR & 59.4 & 46.2 & 44.3 & 37.0 \\
\hline
\end{tabular}

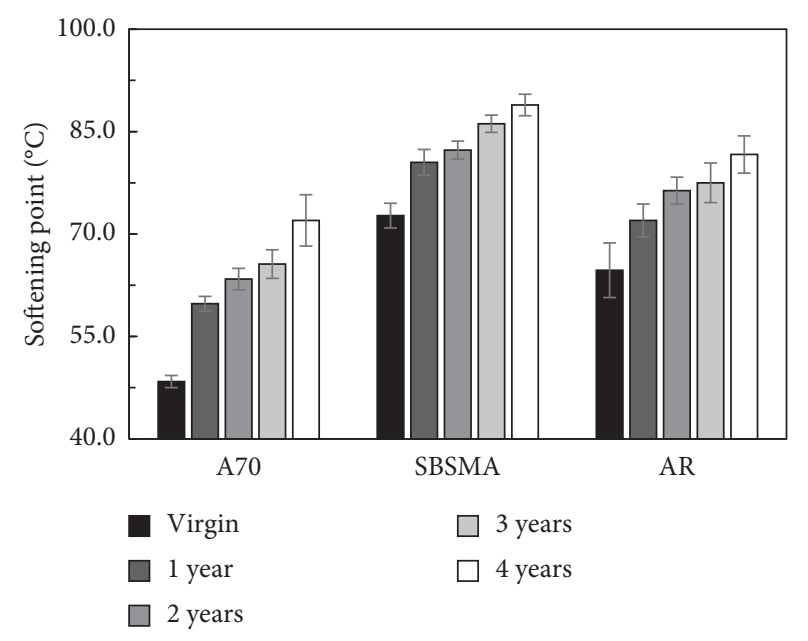

FIGURE 4: Softening point comparison of asphalts with different weathering times.

of the asphalt at various weathering times. The data in Table 5 show that brittle fracturing occurs in neat asphalt A70 and that in the modified asphalt SBSMA, the ductility test after natural-weather aging for 1 year. Ductility data are at value 0 . The ductility dates of SBS-modified asphalt are $11.6\left(10^{\circ} \mathrm{C}\right)$ and $0\left(5^{\circ} \mathrm{C}\right)$ after weathering for 1 year. In subsequent experiments, when the test temperature is increased to $10^{\circ} \mathrm{C}$, brittle fracture still occurs in asphalt A70 and SBSMA, while the ductility value of rubber asphalt continues to be measurable. This finding indicates that rubber asphalt has good ductility under natural weathering conditions.
TABLE 4: Change rate of softening point.

\begin{tabular}{lcccc}
\hline \multirow{2}{*}{ Asphalt } & \multicolumn{4}{c}{ Change rate of softening point (\%) } \\
& 1 year & 2 years & 3 years & 4 years \\
\hline A70 & 23.6 & 31.0 & 35.5 & 48.8 \\
SBSMA & 10.7 & 13.2 & 18.5 & 22.3 \\
AR & 11.3 & 18.0 & 19.8 & 26.2 \\
\hline
\end{tabular}

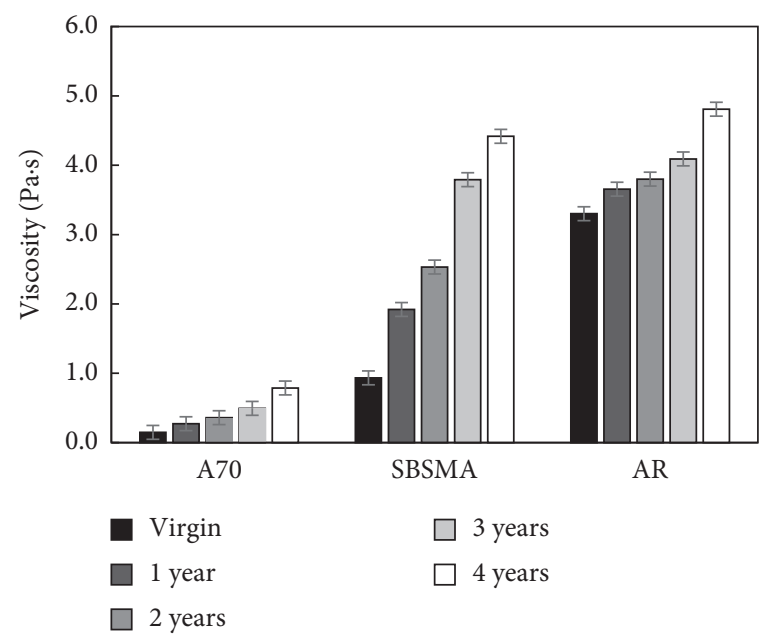

FIgURe 5: Viscosity of asphalt with different weathering time.

TABLE 5: Viscosity change rate of asphalt.

\begin{tabular}{lcccc}
\hline \multirow{2}{*}{ Asphalt } & \multicolumn{4}{c}{ Viscosity increase rate (\%) } \\
& 1 year & 2 years & 3 years & 4 years \\
\hline A70 & 84.5 & 143.2 & 233.8 & 432.4 \\
SBSMA & 105.6 & 171.1 & 306.0 & 372.9 \\
AR & 10.7 & 15.1 & 23.9 & 45.7 \\
\hline
\end{tabular}

The above analysis suggests that natural weathering has a significant impact on the basic physical properties of asphalt. After 4 years of aging, the penetration, softening point, viscosity, and ductility of asphalt exhibited different degrees of attenuation; the aging in the first year has the most significant effect. The performance of rubber asphalt is relatively stable, and base asphalt $\mathrm{A} 70$ has a high aging susceptibility.

Asphalt will suffer from a series of complex physiochemical reactions in the natural environment, such as volatilization, oxidation, and condensation. These reactions will happen in asphalt, and they make it even harder and more brittle. Oxidation plays an important role in asphalt performance. According to the research of Zhao et al. [16], when the asphalt is exposed to the natural environment, oxidation will occur and lead to an increase in its carbonyl and sulfoxide content. The change of the carbonyl index (CI) agrees with the obvious change in rheological properties. Meanwhile, the increase of carbonyl content is less in SBSMA, compared with that of neat asphalt. This indicates that SBSMA is, in terms of oxidation, the superior of neat asphalt in regard to the resistance to the aging process. The 
TABLE 6: Ductility dates of asphalt.

\begin{tabular}{lccccccc}
\hline & \multicolumn{8}{c}{ Ductility $(\mathrm{cm})$} \\
Asphalt & \multicolumn{2}{c}{ Virgin } & \multicolumn{2}{c}{1 year } & 2 years & 3 years & 4 years \\
& $10^{\circ} \mathrm{C}$ & $5^{\circ} \mathrm{C}$ & $10^{\circ} \mathrm{C}$ & $5^{\circ} \mathrm{C}$ & $10^{\circ} \mathrm{C}$ & $10^{\circ} \mathrm{C}$ & $10^{\circ} \mathrm{C}$ \\
\hline A70 & 37.3 & - & 0 & - & 0 & 0 & 0 \\
SBSMA & 48.7 & 28.5 & 11.6 & 0 & 0 & 0 & 0 \\
AR & - & 15.0 & - & 5.1 & 6.3 & 5.5 & 3.3 \\
\hline
\end{tabular}

higher viscosity of SBSMA results in the slower flow of the asphalt, which reduces the chance of oxidation. This leads to a change in the physical properties of base asphalt, more so than in that of SBS-modified asphalt. The carbonyl content increase is lesser in crumb-rubber-modified asphalt than in SBSMA [2]. This indicates that the aging resistance of AR is superior to that of SBSMA. The change rates of carbonyl content are also in agreement with the change ratio of rheological properties. This is consistent with the research findings outlined in this paper. That is, rubber asphalt has superior aging resistance, and base asphalt A70 has high aging susceptibility.

3.2. Cohesive and Adhesive Performance. The contact angle values of asphalt with distilled water, formamide, glycerol, and ethylene glycol reagents are measured by sessile drop testing. The surface energy components of asphalt can be obtained by equation (5), a calculation of the relationship between contact angle and surface free energy. Table 7 lists the surface free energy components of the asphalt with various natural weathering times.

The comparative analysis of the data in Table 7 indicates that the surface free energy of base asphalt A70 increases with aging time, and the change rate reaches $16.9 \%$ after 4 years of aging. However, the surface free energy of SBSMA and AR first increases, reaches the maximum value after the second year of aging, and then decreases gradually in the next 2 years. Asphalt is composed mainly of nonpolar hydrocarbons that cause the dispersive component of asphalt to become far greater than the polarity component. However, the existence of polar chemical groups in asphalt, such as in some functional groups that include carbonyl $(\mathrm{C}=\mathrm{O})$ and sulfoxide $(\mathrm{S}=\mathrm{O})$, has an important role in asphalt performance. After natural weathering, the change characteristics of the polar component of surface energy are also significant. The polarity component of A70 asphalt decreases with aging time, whereas the polar component of the two other kinds of modified asphalt reaches the lowest value after 3 years of aging and increases in the 4 th year. The influence of aging on asphalt composition and the relationship between asphalt chemical composition and surface free energy need further study.
TABLE 7: Surface free energy components of asphalt.

\begin{tabular}{lcccc}
\hline Asphalt & $\begin{array}{c}\text { Aging time } \\
\text { (year) }\end{array}$ & $\gamma\left(\mathrm{mJ} \cdot \mathrm{m}^{-2}\right)$ & $\begin{array}{c}\gamma^{d} \\
\left(\mathrm{~mJ} \cdot \mathrm{m}^{-2}\right)\end{array}$ & $\begin{array}{c}\gamma^{p} \\
\left(\mathrm{~mJ} \cdot \mathrm{m}^{-2}\right)\end{array}$ \\
\hline \multirow{4}{*}{ A70 } & 0 & 14.49 & 11.95 & 2.55 \\
& 1 & 16.17 & 14.69 & 1.48 \\
& 2 & 16.20 & 14.72 & 1.48 \\
& 3 & 16.52 & 15.35 & 1.17 \\
& 4 & 16.94 & 15.86 & 1.08 \\
\hline \multirow{3}{*}{ SBSMA } & 0 & 14.48 & 11.52 & 2.97 \\
& 1 & 15.68 & 13.80 & 1.87 \\
& 2 & 17.16 & 15.82 & 1.34 \\
& 3 & 17.00 & 15.88 & 1.12 \\
AR & 4 & 16.92 & 15.78 & 1.15 \\
\hline & 0 & 15.63 & 13.60 & 2.03 \\
& 1 & 17.24 & 15.72 & 1.52 \\
& 2 & 17.44 & 16.17 & 1.27 \\
& 3 & 17.15 & 15.90 & 1.25 \\
& 4 & 16.77 & 15.16 & 1.61 \\
\hline
\end{tabular}

The work of cohesion is defined as the energy consumed when a homogeneous material is divided into two new surfaces. Value can be calculated from the surface free energy on the following equation:

$$
W_{\text {cohesion }}=2 \gamma_{\text {asphalt }}=2\left(\gamma_{\text {asphalt }}^{d}+\gamma_{\text {asphalt }}^{p}\right),
$$

where $W_{\text {cohesion }}$ is the work of cohesion, $\gamma_{\text {asphalt }}$ is the surface free energy of asphalt, $\gamma_{\text {asphalt }}^{d}$ is the dispersive component, and $\gamma_{\text {asphalt }}^{p}$ is the polarity component.

Figure 6 presents the work of asphalt cohesion with different natural weathering times. The work of cohesion has the same change trend as the surface free energy of asphalt. The cohesive work of base asphalt increases gradually with aging time, and the cohesive work of modified asphalt rises initially, reaches the maximum value after the second year of aging, and then decreases gradually in the next two years. The results show that the presence of SBS and rubber powder transforms the change trend of the cohesive work of the matrix asphalt. This phenomenon causes the modified asphalt to become more prone to cohesive failure after longterm natural weathering.

The work of adhesion can be observed in the decrease in free energy when a two-phase material is separated. In dry conditions, the value can be calculated from the surface free energy according to the following equation [25]:

$$
\begin{aligned}
W_{\text {adhesion,dry }} & =\gamma_{\text {asphalt }}+\gamma_{\text {aggregate }}-\gamma_{\text {asphalt-aggregate }} \\
& =2 \sqrt{\gamma_{\text {asphalt }}^{d} \gamma_{\text {aggregate }}^{d}}+2 \sqrt{\gamma_{\text {asphalt }}^{p} \gamma_{\text {aggregate }}^{p}} .
\end{aligned}
$$

Under water conditions, the work of adhesion between asphalt and aggregate can be formulated as follows [25]: 


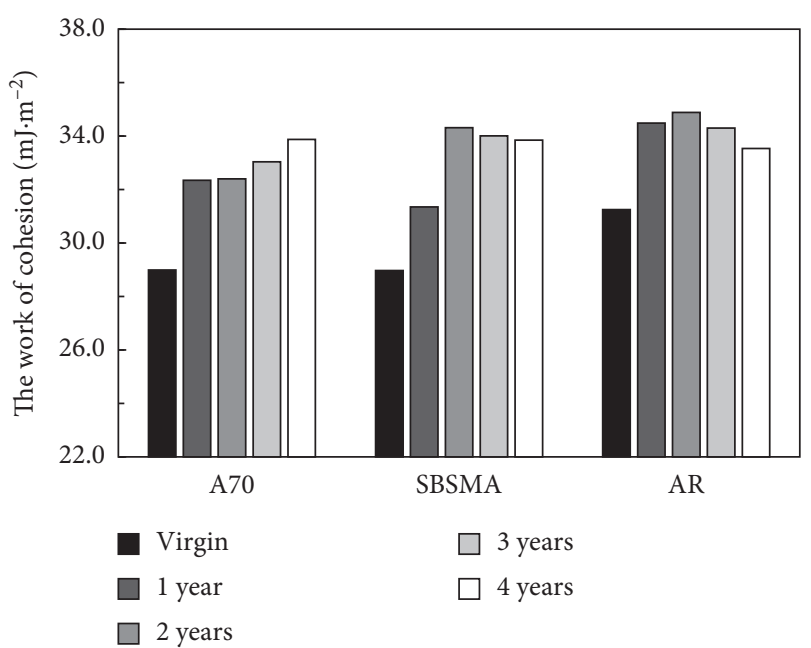

Figure 6: Work of cohesion of asphalt.

TABLE 8: Surface free energy components of aggregates.

\begin{tabular}{lccc}
\hline Aggregates & $\gamma\left(\mathrm{mJ} \cdot \mathrm{m}^{-2}\right)$ & $\gamma^{d}\left(\mathrm{~mJ} \cdot \mathrm{m}^{-2}\right)$ & $\gamma^{p}\left(\mathrm{~mJ} \cdot \mathrm{m}^{-2}\right)$ \\
\hline Limestone & 47.1 & 24.3 & 22.8 \\
Basalt & 58.5 & 30.1 & 28.4 \\
Granite & 57.3 & 16.9 & 40.4 \\
\hline
\end{tabular}

$$
\begin{aligned}
W_{\text {adhesion,wet }}= & \gamma_{\text {asphalt-water }}+\gamma_{\text {aggregate-water }}-\gamma_{\text {asphalt-aggregate }} \\
= & 2\left(\gamma_{\text {water }}+\sqrt{\gamma_{\text {aggregate }}^{d} \cdot \gamma_{\text {asphalt }}^{d}}+\sqrt{\gamma_{\text {aggregate }}^{p} \cdot \gamma_{\text {asphalt }}^{p}}\right. \\
& \left.-\sqrt{\gamma_{\text {asphalt }}^{d} \cdot \gamma_{\text {water }}^{d}}-\sqrt{\gamma_{\text {asphalt }}^{p} \cdot \gamma_{\text {water }}^{p}}-\sqrt{\gamma_{\text {aggregate }}^{d} \cdot \gamma_{\text {water }}^{d}}-\sqrt{\gamma_{\text {aggregate }}^{p} \cdot \gamma_{\text {water }}^{p}}\right),
\end{aligned}
$$

where $W_{\text {adhesion,dry }}$ is the work of adhesion under dry conditions, $W_{\text {adhesion,wet }}$ is the work of adhesion under water condition, $\gamma_{\text {aggregate }}$ is the surface free energy of the aggregate, $\gamma_{\text {aggregate }}^{d} \gamma_{\text {aggregate }}^{p}$ are the dispersive and polarity components of aggregate surface free energy, $\gamma_{\text {asphalt-water }}$ is the interface of the free energy between asphalt and water, $\gamma_{\text {aggregate-water }}$ is the interface of free energy between aggregate and water, and $\gamma_{\text {asphalt-aggregate }}$ is the interface free energy between aggregate and asphalt.

The surface free energy components of aggregates are listed in Table 8. The surface free energy of limestone is lower than those of basalt and granite. The dispersion components of limestone and basalt are slightly larger than that of the polar component, whereas the dispersion component of granite is much smaller than that of the polar component. According to equations (7) and (8), the adhesion work of asphalt and aggregate under dry and water conditions can be obtained.

The work of adhesion between asphalt and aggregate under dry conditions is shown in Figure 7. The results in this study show that the surface energy of the aggregate is the main factor that affects adhesive performance. Under dry conditions, the work of adhesion between asphalt and basalt is maximal and that of granite is minimal. This result indicates that the adhesive property and antispalling ability of asphalt and basalt are better than that of granite. Compared with the cohesion work of asphalt, as shown in Figure 6, the adhesion work of asphalt and the three kinds of aggregate is greater than that of asphalt cohesion work under dry conditions. This indicates that the cohesive failure of the asphalt mixture will occur preferentially when subjected to external forces under dry conditions.

However, with the deepening of the natural weathering of asphalt, the work of adhesion between asphalt and aggregate under dry conditions shows a decreasing trend, and the numerical value changes slightly. Nevertheless, its influence on the adhesion of granite is relatively significant. Natural weathering reduces the adhesive properties of asphalt.

The presence of moisture is an important factor in the acceleration of the water damage of the asphalt mixture. The work of adhesion between asphalt and aggregate under water conditions is shown in Figure 8. The adhesion work of asphalt and aggregate in a water environment is significantly reduced and is less than the cohesion work of asphalt in dry conditions. The presence of water leads to the poor adhesive strength of asphalt and aggregate. When moisture damage occurs, asphalt will tend to peel off from the surface of the aggregate, rather than fail cohesively. Comparing the three aggregates, the order of adhesion work, from the greatest to 


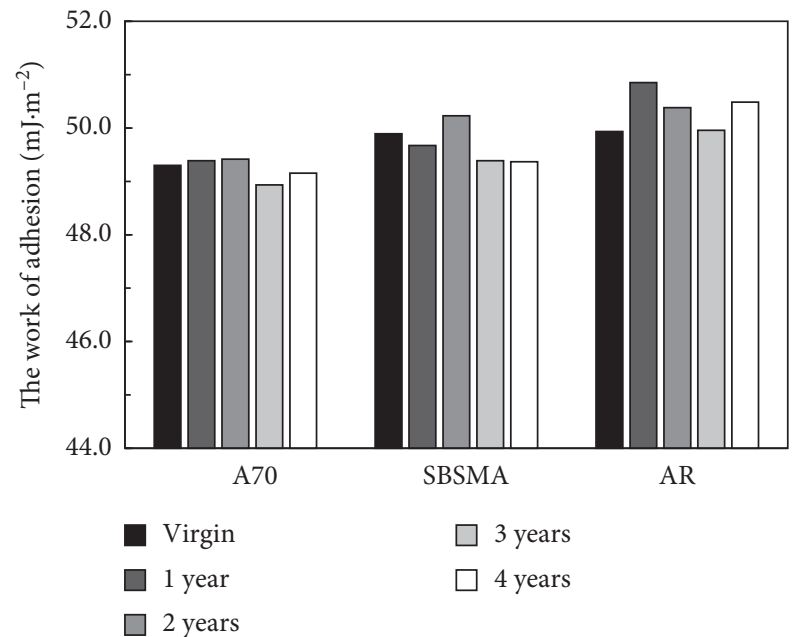

(a)

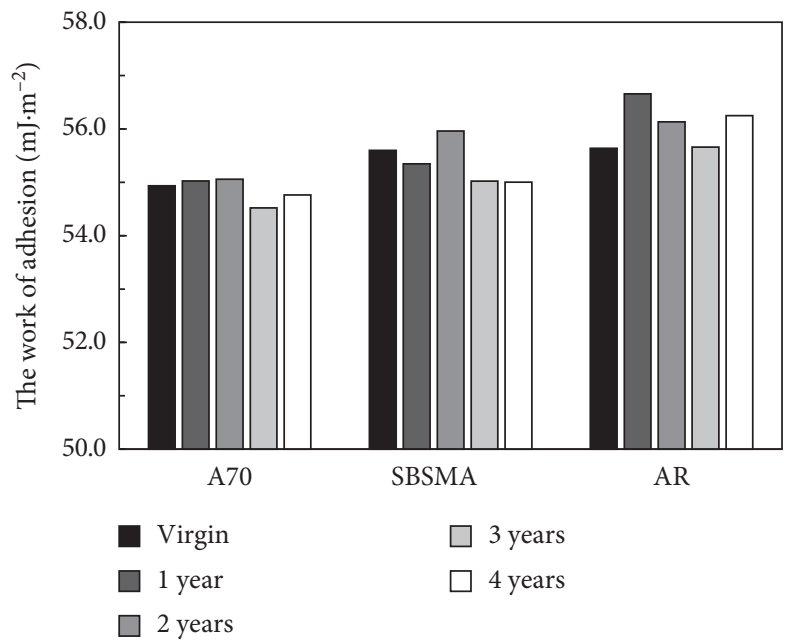

(b)

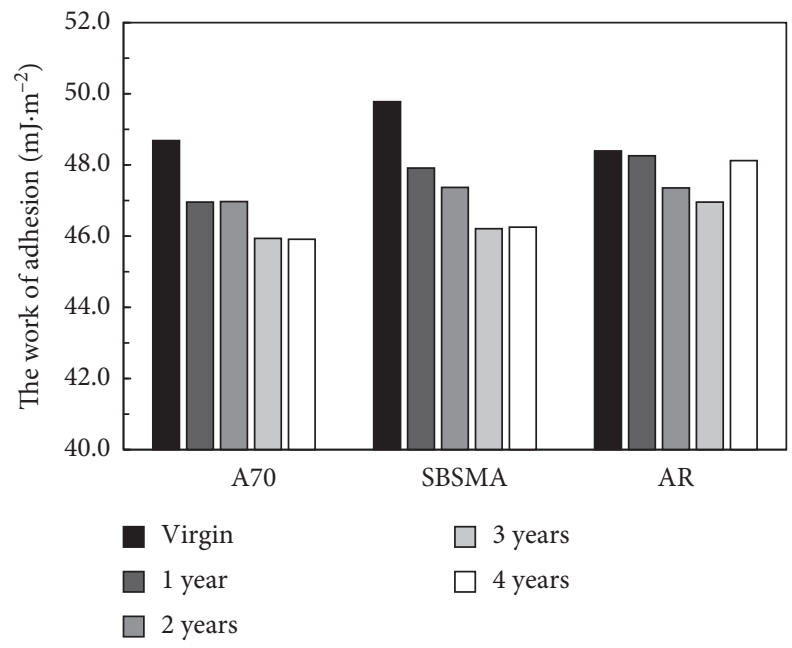

(c)

FiguRE 7: Work of adhesion between asphalt and (a) limestone, (b) basalt, and (c) granite under dry conditions.

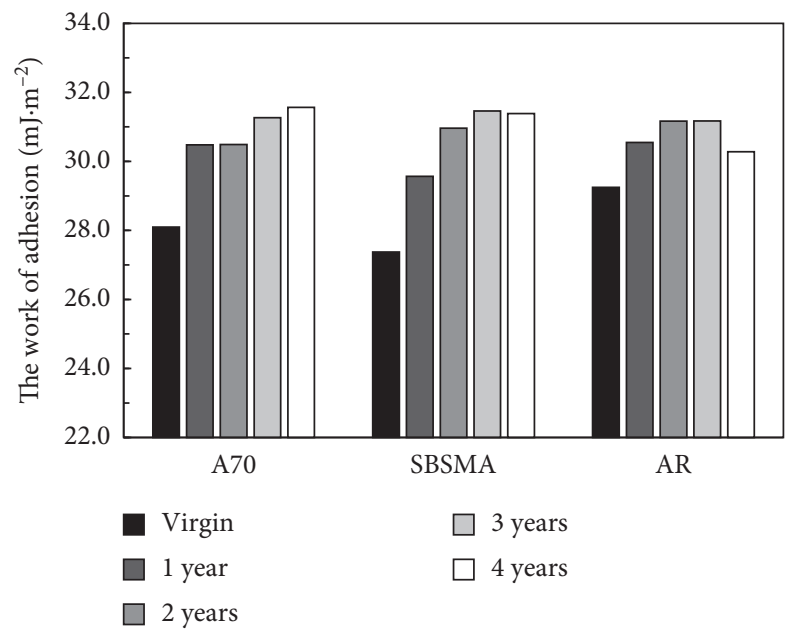

(a)

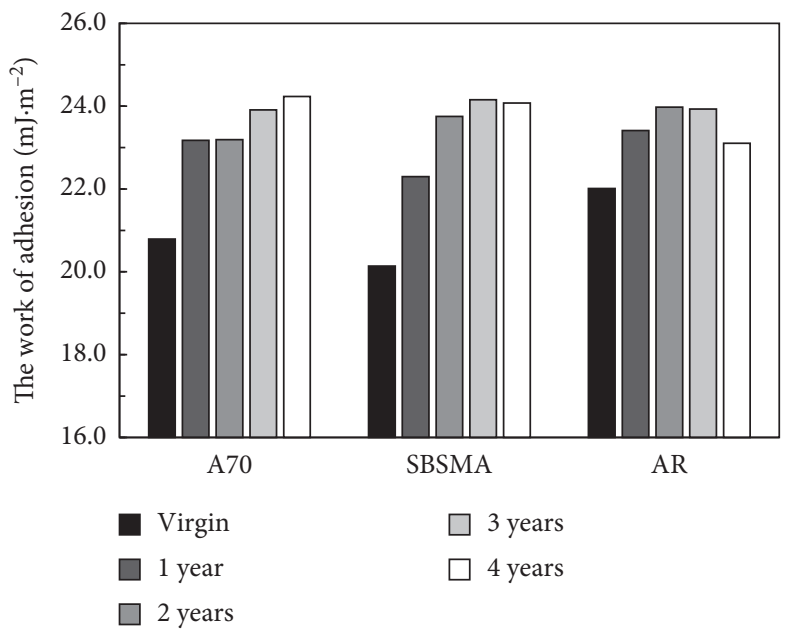

(b)

Figure 8: Continued. 


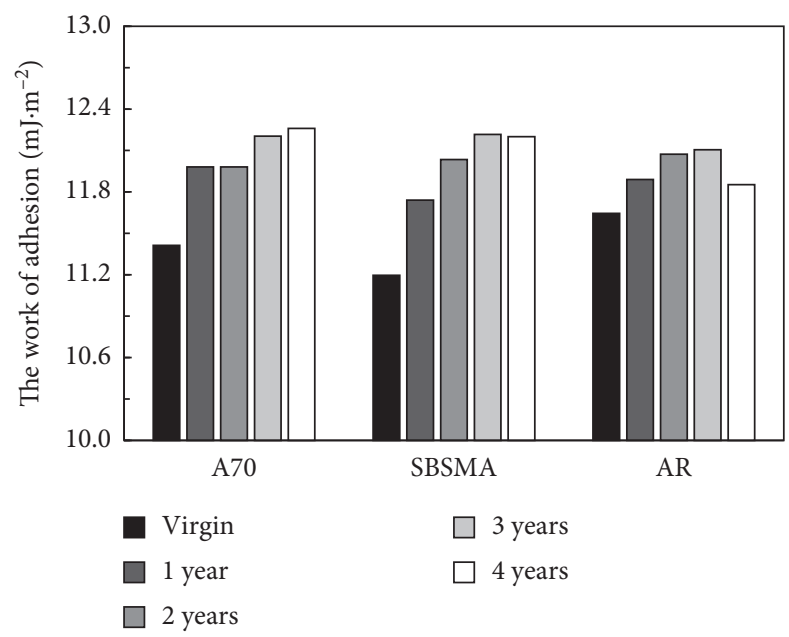

(c)

FIGURE 8: Work of adhesion with asphalt and (a) limestone, (b) basalt, and (c) granite under water condition.

the least, is limestone, basalt, and granite. Asphalt is a kind of weak acid material that easily adheres to alkaline aggregates. However, granite is an acid stone, and the adhesion work of granite and asphalt is less than those of the two other aggregates, regardless of whether water is present. Therefore, from the point of view of adhesion work theory, granite and asphalt have a poor adhesive performance.

Furthermore, under water conditions, the work of adhesion with neat asphalt A70 increases gradually with aging time and that of modified asphalt increases initially and then decreases after 4 years of natural aging. This observation is consistent with the change trend of the surface free energy of asphalt but different from that under dry conditions.

Asphalt will suffer from a series of complex physical and chemical reactions in the natural environment because of sunlight, heat, oxygen, and moisture. When the asphalt is exposed to the natural environment, some light components will volatilize. The components of asphalt will also change in sequence. That is, the aromatics will be converted into the resin, the resin will be transformed into asphaltene, and the asphaltene can be transformed also into a toluene insoluble substance, because of molecular association and condensation. Carbonyl sulfoxide and other compound groups will be produced because of the reaction of the functional groups to oxygen.

Overall, the molecular weight of asphalt increases. However, the aging process of modified asphalt is more complex. The interaction between SBS copolymer, which has a higher molecular weight, and neat asphalt causes the modified asphalt to have better oxidation resistance. The deepening of the aging of SBS will degrade into small molecular substances [16]. Similarly, in rubber asphalt, the rubber powder will pyrolyze, and the pyrolysis products will oxidize and condense, thereby making the aging mechanism of rubber asphalt more complex. This phenomenon may be the reason for the change trend of surface energy. Alternately, the adhesion work of modified asphalt differs from that of neat asphalt.
The above analysis of the adhesion of asphalt and the aggregates shows that the property of each aggregate is the main factor in adhesion, whereas the influences of asphalt properties and natural weathering are relatively mild. For granite, whether water is present or absent, adhesion to asphalt is less likely than it is for the two other aggregates. Compared with limestone, basalt has better adhesion under dry conditions, whereas limestone has better adhesion when water is present. The presence of moisture changes cohesive failure into adhesion failure between asphalt and aggregate. The adhesive performance indicator is relatively independent of basic physical properties and needs further research.

\section{Conclusions}

The neat asphalt A70, SBS-modified asphalt SBSMA, and crumb-rubber-modified asphalt AR were exposed to outdoor conditions for four years, for natural weathering. The physical properties, surface free energy, and adhesive properties of asphalt were evaluated. The following conclusions can be drawn:

(1) Natural weathering causes asphalt to harden and become brittle, thereby reducing penetrability and increasing softening points and viscosity. After four years of aging, the residual penetration ratio of asphalt is only $18.5 \% \sim 37.0 \%$, and the increased rates of the softening point reach $22.3 \% \sim 48.8 \%$. Meanwhile, aging in the first year has the most significant effect.

(2) The rubber asphalt AR has superior aging resistance, and base asphalt A70 has a high aging susceptibility. The viscosity change rate of rubber asphalt AR is only $45.7 \%$ after four years of aging, whereas viscosity increases for neat asphalt and SBS-modified asphalt reaches as high as $432.4 \%$ and $372.9 \%$. After four years of aging, the ductility value of rubber asphalt is still greater than $3 \mathrm{~cm}$, while the other two kinds of asphalt have deteriorated to a state of brittle fracture. 
(3) The surface free energy of neat asphalt gradually increases with aging time, while that of modified asphalt increases initially, reaches the maximum value after the second year of aging, and then decreases gradually in the next two years. The presence of SBS and rubber powder transform the change trend of the surface free energy of neat asphalt.

(4) The work of adhesion can evaluate adhesive properties effectively, and the surface free energy of aggregate and moisture are the main factors that affect adhesive performance. Under dry conditions, the adhesion work is about $50 \mathrm{~mJ} \cdot \mathrm{m}^{-2}$, but when moisture is present, the adhesion work of limestone, basalt, and granite decrease to about $30 \mathrm{~mJ} \cdot \mathrm{m}^{-2}$, $23 \mathrm{~mJ} \cdot \mathrm{m}^{-2}$, and $12 \mathrm{~mJ} \cdot \mathrm{m}^{-2}$, respectively. These are less than the cohesion work of asphalt, which is about $33 \mathrm{~mJ} \cdot \mathrm{m}^{-2}$. This means that the presence of moisture changes cohesive failure into adhesive failure.

Asphalt will suffer from a series of complex physical and chemical reactions in the natural environment because of sunlight, heat, oxygen, and moisture. The change in asphalt chemical components should be studied further to discover the natural weathering law that should guide the production and application of asphalt.

\section{Data Availability}

The data used to support the findings of this study are available from the corresponding author upon request.

\section{Conflicts of Interest}

The authors declare that they have no conflicts of interest.

\section{Acknowledgments}

This research was supported by the Special Fund of Chinese Central Government for Basic Scientific Research Operations in Commonweal Research Institutes (no.2018-9016).

\section{References}

[1] S. Xu, A. García, J. Su et al., "Self-healing asphalt review: from idea to practice," Advanced Materials Interfaces, vol. 17, no. $17,2018$.

[2] Q. Wang, S. Li, X. Wu, S. Wang, and C. Ouyang, "Weather aging resistance of different rubber modified asphalts," Construction and Building Materials, vol. 106, no. 106, pp. 443-448, 2016.

[3] W. D. Fernández-Gómez, H. A. Rondón Quintana, C. E. Daza, and F. A. Reyes Lizcano, "The effects of environmental aging on Colombian asphalts," Fuel, vol. 115, no. 115, pp. 321-328, 2014.

[4] R. Moraes, R. Velasquez, and H. Bahia, "Using bond strength and surface energy to estimate moisture resistance of asphaltaggregate systems," Construction and Building Materials, vol. 130, no. 130, pp. 156-170, 2017.

[5] M. Koc and R. Bulut, "Assessment of a sessile drop device and a new testing approach measuring contact angles on aggregates and asphalt binders," Journal of Materials in Civil Engineering, vol. 26, no. 3, pp. 391-398, 2014.

[6] A. W. Hefer, A. Bhasin, and D. N. Little, "Bitumen surface energy characterization using a contact angle Approach," Journal of Materials in Civil Engineering, vol. 18, no. 6, pp. 759-767, 2006.

[7] F. Wang, Y. Xiao, P. Cui et al., "Correlation of asphalt performance indicators and aging Degrees: a review," Construction and Building Materials, vol. 250, no. 250, Article ID 118824, 2020.

[8] J. Lin, J. Hong, J. Liu, and S. Wu, "Investigation on physical and chemical parameters to predict long-term aging of asphalt binder," Construction and Building Materials, vol. 122, no. 122, pp. 753-759, 2016.

[9] D. Zhang, H. Zhang, and C. Shi, "Investigation of aging performance of SBS modified asphalt with various aging methods," Construction and Building Materials, vol. 145, no. 145, pp. 445-451, 2017.

[10] S.-J. Lee, S. N. Amirkhanian, K. Shatanawi, and K. W. Kim, "Short-term aging characterization of asphalt binders using gel permeation chromatography and selected Superpave binder tests," Construction and Building Materials, vol. 22, no. 11 , pp. 2220-2227, 2008 .

[11] P. Li, Z. Ding, Z. Zhang, and D. Zhang, "Comparative evaluation of laboratory and field ageing of asphalt binder using a non-linear differential model," Road Materials and Pavement Design, vol. 17, no. 2, pp. 434-445, 2016.

[12] V. Mouillet, F. Farcas, E. Chailleux et al., "Evolution of bituminous mix behaviour submitted to UV rays in laboratory compared to field exposure," Materials and Structures, vol. 47, no. 8, pp. 1287-1299, 2014.

[13] J. Tu, J. Yuan, C. O. Bao et al., "Study on ultraviolet radiation aging of road asphalt and SBS modified asphalt," Petroleum Asphalt, vol. 22, no. 6, pp. 43-47, 2008.

[14] P. Xiao, M. P. Wu, and D. A. Jiang, "Study on properties of ultraviolet aged rubber asphalt," Journal of Nanjing University of Aeronautics \& Astronautics, vol. 45, no. 1, pp. 152-156, 2013.

[15] C. Z. Zhu, H. L. Zhang, D. M. Zhang et al., "Influence of base asphalt and SBS modifier on the weathering aging behaviors of SBS modified asphalt," Journal of Materials In Civil Engineering, vol. 30, no. 3, Article ID 04017306, 2018.

[16] X. Zhao, S. Wang, Q. Wang, and H. Yao, "Rheological and structural evolution of SBS modified asphalts under natural weathering," Fuel, vol. 184, no. 184, pp. 242-247, 2016.

[17] X. Yu, N. A. Burnham, R. B. Mallick, and M. Tao, "A systematic AFM-based method to measure adhesion differences between micron-sized domains in asphalt binders," Fuel, vol. 113, pp. 443-447, 2013.

[18] J. Yu, Z. Dai, J. Shen, H. Zhu, and P. Shi, “Aging of asphalt binders from weathered asphalt mixtures compared with a SHRP process," Construction and Building Materials, vol. 160, no. 160, pp. 475-486, 2018.

[19] S.-H. Kim, J.-H. Jeong, and N. Kim, "Use of surface free energy properties to predict moisture damage potential of Asphalt concrete mixture in cyclic loading condition," KSCE Journal of Civil Engineering, vol. 7, no. 4, pp. 381-387, 2003.

[20] C. J. van Oss, M. K. Chaudhury, and R. J. Good, "Monopolar surfaces," Advances in Colloid and Interface Science, vol. 28, no. 1, pp. 35-64, 1987.

[21] D. Y. Kwok and A. W. Neumann, "Contact angle measurement and contact angle interpretation," Advances in Colloid and Interface Science, vol. 81, no. 3, pp. 167-249, 1999. 
[22] C. J. Van, "Use of the combined Lifshitz-van der Waals and Lewis acid-base approaches in determining the apolar and polar contributions to surface and interfacial tensions and free energies," Journal of Adhesion Science and Technology, vol. 16, no. 6, pp. 669-677, 2002.

[23] D. K. Owens and R. C. Wendt, "Estimation of the surface free energy of polymers," Journal of Applied Polymer Science, vol. 13, no. 13, pp. 1741-1747, 1969.

[24] F. M. Fowkes, "Additivity of intermolecular forces at interfaces. I. determination of the contribution to surface and interfacial tensions of dispersion forces in various liquids 1 ," The Journal of Physical Chemistry, vol. 67, no. 12, pp. 25382541, 1963.

[25] Y. Tan and M. Guo, "Using surface free energy method to study the cohesion and adhesion of asphalt mastic," Construction and Building Materials, vol. 47, no. 47, pp. 254-260, 2013. 